\title{
Billiards on almost integrable polyhedral surfaces
}

\author{
E. GUTKIN \\ Columbia University, New York, USA and Max-Planck-Institute für Mathematik, \\ Bonn, West Germany $\dagger$
}

Received 16 November 1983 and revised 9 April 1984)

\begin{abstract}
The phase space of the geodesic flow on an almost integrable polyhedral surface is foliated into a one-parameter family of invariant surfaces. The fiow on a typical invariant surface is minimal. We associate with an almost integrable polyhedral surface its holonomy group which is a subgroup of the group of motions of the Euclidean plane. We show that if the holonomy group is discrete then the flow on an invariant surface is ergodic if and only if it is minimal.
\end{abstract}

\section{Introduction}

Geodesic flows on Euclidean polyhedra is an old subject that goes as far back as 1906 (see [10] and a related paper [8]). An example of such a flow is the motion of a billiard ball inside a polygon. If the angles of the polygon are rational multiples of $\pi$, the direction of any geodesic takes only a finite number of values as time varies. Fixing these values one obtains invariant 'surfaces' of the billiard flow and the induced flow on the typical invariant surface is minimal ([13]). It is not known whether the flow is typically ergodic (with respect to the invariant Lebesgue measure). The general expectation is that the answer is yes. $\neq$ This question is related to the question of whether the typical interval exchange transformation is ergodic, where the answer is positive ([5], [12]).

For arbitrary polyhedral surfaces the condition that vertex angles are $\pi$-rational does not insure the existence of invariant surfaces for the geodesic flow. One needs an extra condition that a certain holonomy group is finite. We call surfaces satisfying this condition almost integrable because the geodesic flow which is a Hamiltonian system with two degrees of freedom has an additional integral of motion which 'almost commutes' with the Hamiltonian.

In $\S 1$ we associate with any almost integrable surface $S$ a Riemann surface $R$ in a purely geometric way. The genus of $R$ is determined by the vertex angles of $S$ (formulae (8)-(11)). The main tool is the developing map of the universal covering of $S$ onto the complex plane. The typical invariant surface of the geodesic flow of $S$ (which we call the billiard flow) is isomorphic to $R$. Topology of the foliation of the phase space of the billiard flow into invariant surfaces is discussed in [4].

† Current address: University of Southern California, Los Angeles, U.S.A.

$\ddagger$ Added in proof: S. Kerckhoff, H. Masur and J. Smillie have recently proved that the flow is typically uniquely ergodic. 
In $\S 2$ we show that the billiard flow of $S$ is equivalent to a family of $b_{\theta}^{t}, 0 \leq \theta<2 \pi$, of flows on $R$ ( $b_{\theta}^{t}$ is the billiard flow in direction $\theta$ ). They are pulled back by the developing map from the linear flows $l_{\theta}^{\prime}$ on $\mathbb{C}$. At the end of the section we extend to $b_{\theta}^{t}$ the minimality results of [13] and [3].

In $\S 3$ we consider a class of almost integrable surfaces $S$ given essentially by the condition that the full holonomy group of $S$ is discrete. For these surfaces, as theorem 3 shows, the billiard flow $b_{\theta}^{t}$ is minimal if and only if it is uniquely ergodic (which is false for general almost integrable billiards). Moreover in this case $b_{\theta}^{t}$ extends the linear flow $l_{\theta}^{t}$ on a certain torus intrinsically defined by $S$, thus $b_{\theta}^{t}$ is minimal if and only if $\theta$ is an irrational direction. If $\theta$ is irrational the discrete spectrum of $b_{\theta}^{t}$ coincides with the spectrum of $l_{\theta}^{t}$, i.e. $b_{\theta}^{t}$ is weakly mixing modulo $l_{\theta}^{t}$. It is reasonable to expect that for almost integrable polyhedral surfaces outside of this class the billiard flow $b_{\theta}^{t}$ is typically weakly mixing.

Some results of the paper generalize to billiards on higher dimensional polyhedra which will be discussed elsewhere. It is worth mentioning that almost integrable billiards (both classical and quantum) are of interests to physicists (cf. [7], [1]).

I would like to thank A. Katok and W. Veech for useful consultations. This work was partially supported by NSF Grant MCS 8101739 .

\section{Developing map}

By Euclidean polygon we mean a closed bounded polygon $P$ in $\mathbb{C}$ such that its interior $P \backslash \partial P$ is connected. If $P$ has more than one connected component we say that $P$ has obstacles. If an obstacle has two adjacent sides with angle $2 \pi$ between them we say that $P$ has a slit (see figure 1).

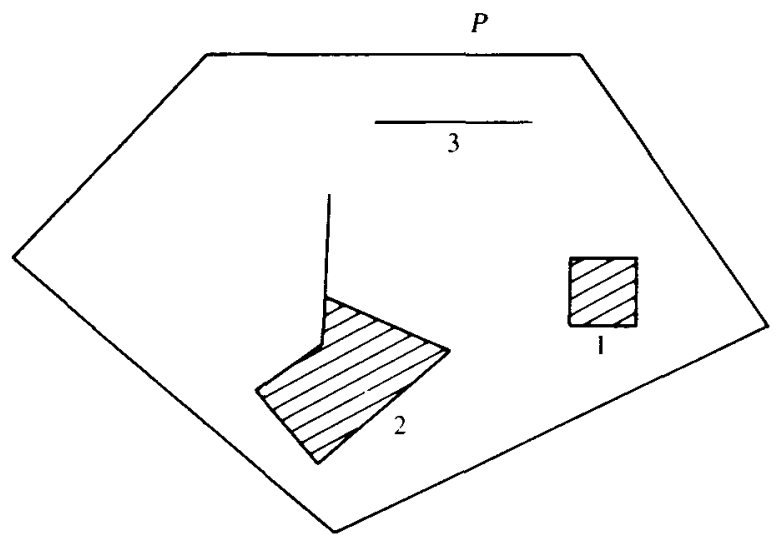

FIGURE 1. The shaded regions are obstacles. Obstacle 2 has a slit and obstacle 3 is just a slit.

A Euclidean polyhedron $S$ (of dimension 2) is a collection of Euclidean polygons with some sides identified by isometries. These polygons, their sides and vertices are the faces, edges and vertices of $S$. A Euclidean polyhedron has a natural topology.

Definition 1. A polyhedral surface $S$ is a connected Euclidean polyhedron homeomorphic to a topological surface.

If a polyhedron $S$ has vertices with an infinite number of adjacent faces then $S$ cannot be a polyhedral surface. We say that $S$ is a polyhedral surface with vertices 
at infinity if $S$ with those vertices punctured becomes a topological surface. Henceforth polyhedron will mean a polyhedral surface possibly with vertices at infinity. A polyhedral surface is closed if it has no boundary, $\partial S=\varnothing$. If $\partial S \neq \varnothing$ two copies of $S$ glued along the boundary make a closed polyhedral surface $d S$ called the doubling of $S$.

There is a canonical complex structure on any oriented polyhedral surface. Assume first that $\partial S=\varnothing$. Every face and every edge of $S$ define a coordinate patch in an obvious way. Let $A$ be a vertex of $S$ and let $P_{1}, \ldots, P_{n}$ be the adjacent faces in an orientation preserving order. Let $\alpha_{1}, \ldots, \alpha_{n}$ be their respective angles. The sum $\alpha=\alpha_{1}+\cdots+\alpha_{n}$ is called the angle of $A$. Cut $U=P_{1} \cup \cdots \cup P_{n}$ along an edge $b$ and unfold it on $\mathbb{C}$ so that $A$ goes into 0 and $b$ goes into the positive real axis. Let $z$ be the complex coordinate in $\mathbb{C}$. Then $u=z^{2 \pi / \alpha}$ is a well defined coordinate in $U$. It is straightforward to check that the transition functions of the covering are complex analytic and that the complex structure thus defined does not depend on the choices made. If $\partial S \neq \varnothing$ the imbedding $S \subset d S$ defines the complex structure on $S$. Thus any polyhedral surface is a Riemann surface.

Polyhedra $S_{1}, S_{2}$ are isomorphic if there is a continuous invertible mapping $f: S_{1} \rightarrow S_{2}$ which maps faces isometrically onto faces. Given a polyhedron $S$ one can always draw new edges on the faces of $S$. This operation does not change $S$ essentially.

Definition 2. Polyhedra $S_{1}, S_{2}$ are called equivalent if they can be made isomorphic by adding new edges.

A group $G$ of automorphisms of a polyhedron $S$ acts properly discontinuously if for any face $P \subset S$ there is only a finite number of $g \in G$ such that $g P \cap P \neq \varnothing$. The quotient $S / G$ is naturally a polyhedron.

Definition 3. A mapping $f: S_{1} \rightarrow S_{2}$ of polyhedra is a covering if for any $x \in S_{1}$ there is $S_{1}^{\prime}$ equivalent to $S_{1}$, a subpolyhedron $R$ of $S_{1}^{\prime}$ containing $x$ and a group $G$ acting properly discontinuously on $R$ such that $S_{2}$ is equivalent to $R / G$ and $\left.f\right|_{R}$ coincides with the natural projection $R \rightarrow R / G$.

If $G$ acts on $S$ properly discontinuously the covering $S \rightarrow S / G$ is the regular covering with the group $G$ of deck transformations. The reader should be aware of the fact that coverings of polyhedra are usually branched. If $\partial S \neq \varnothing$ the natural involution of $d S$ defines a regular covering $d S \rightarrow S$ with the group $\mathbb{Z} / 2$ of deck transformations branched at $\partial S \subset d S$.

Let $S$ be a closed polyhedron and let $x_{0}$ be an interior point of a face $P_{0} \subset S$. Consider the set of continuous loops on $S$ starting at $x_{0}$ and avoiding vertires. The set of homotopy classes of these loops endowed with the usual composition becomes a group $\pi_{f}(S)$ called the full fundamental group of $S$. If $\dot{S}$ is the topological surface obtained by puncturing $S$ at the vertices then $\pi_{f}(S)=\pi_{1}(\dot{S})$. Let $\partial S \neq \varnothing$. Imbed $S$ into $d S$ and denote by $S_{0}, S_{1}$ the image and the mirror image of $S$ respectively. Let $x_{1} \in S_{1}$ be the mirror image of $x_{0} \in S_{0}$. To define $\pi_{f}(S)$ we start from the set of piecewise smooth loops transversal to $\partial S$. Any such loop $\gamma$ has a unique lifting $\tilde{\gamma}$ on $d S$ if we agree that $\tilde{\gamma}$ passes from $S_{0}$ to $S_{1}$ or vice versa each time as $\gamma$ bounces off $\partial S$. We say that $\gamma_{1}$ and $\gamma_{2}$ are equivalent if $\tilde{\gamma}_{1}$ and $\tilde{\gamma}_{2}$ are homotopic with fixed ends. The set of equivalence classes with the usual operation is the group $\pi_{f}(S)$. 
There is an obvious exact sequence

$$
1 \rightarrow \pi_{f}(d S) \rightarrow \pi_{f}(S) \rightarrow \mathbb{Z} / 2 \rightarrow 1 .
$$

A natural class of coverings of $S$ is associated with the subgroups of $\pi_{f}(S)$. Let $\partial S=\varnothing$ and let $H \subset \pi_{f}(S)$ be a subgroup. The imbedding $\dot{S} \subset S$ identifies $H$ with a subgroup of $\pi_{1}(\dot{S})$. Let $\dot{S}^{H}$ be the unbranched covering of $\dot{S}$ corresponding to $H$. Filling in the punctures we obtain a closed polyhedron $S^{H}$ and the covering $p_{H}: S^{H} \rightarrow S$. If $\partial S \neq \varnothing$ we define $S^{H}$ for any subgroup $H \subset \pi_{f}(d S)$ to be $(d S)^{H}$ with the projection $p_{H}:(d S)^{H} \rightarrow d S \rightarrow S$. The coverings $p_{H}: S^{H} \rightarrow S$ are branched at the vertices and above $\partial S$.

Proposition 1. Let $S$ be a closed polyhedron and let $H, G$ be subgroups of $\pi_{f}(S)$.

(i) The inclusion $H \subset G$ holds if and only if there is a covering $q: S^{H} \rightarrow S^{G}$ such that the diagram below commutes:

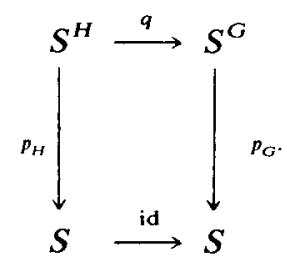

(ii) For any subgroup $H \subset \pi_{f}(S)$ we have $\pi_{f}\left(S^{H}\right)=H$. If $H$ is normal then $p_{H}: S^{H} \rightarrow$ $S$ is a regular covering with the group $\pi_{f}(S) / H$ of deck transformations.

The proof and the generalization to the case $\partial S \neq \varnothing$ are straightforward and are left to the reader.

Definition 4. The covering of $S$ corresponding to the trivial subgroup of $\pi_{f}(S)$ is called the universal covering and is denoted by $\tilde{S}$.

Definition 5. Let $S$ be a closed polyhedron. A mapping $\varphi: S \rightarrow \mathbb{C}$ is called a developing map if it is an isometry on every face of $S$ and if for any edge $b$ there is a neighbourhood $U$ of the interior of $b$ such that $\varphi$ is an isometry on $U$.

A closed polyhedron $S$ is called developable if there exists a developing map $\varphi: S \rightarrow \mathbb{C}$.

Proposition 2. (i) For any closed polyhedron $S$ the following are equivalent:

(a) $S$ is developable;

(b) there exists an isometry $\varphi_{0}: P_{0} \rightarrow \mathbb{C}$ of a face $P_{0}$ which continues to a developing $\operatorname{map} \varphi: S \rightarrow \mathbb{C}$

(c) any isometry $\varphi_{0}: P_{0} \rightarrow \mathbb{C}$ uniquely extends to a developing map $\varphi: S \rightarrow \mathbb{C}$.

(ii) Let $O(\mathbb{C})$ denote the group of isometries of $\mathbb{C}$. Then for any two developing maps $\varphi, \psi$ of $S$ there is a unique $g \in O(\mathbb{C})$ such that $\psi=g \circ \varphi$.

The proof is obvious and is left to the reader. For general polyhedra $S$ the universal covering $\tilde{S}$ is the minimal developable covering of $S$. 
Definition 6. A polyhedral surface $S$ is called rational if any vertex angle of $S$ is $\pi$ times a rational number.

Let $S$ be a closed rational polyhedral surface. Let $A_{i}$, with angles $2 \pi m_{i} / n_{i}\left(m_{i}\right.$ and $n_{i}$ are coprime), $i \in I$, be the vertices of $S$. Denote by $g_{i} \in \pi_{f}(S)$ the equivalence class of a simple loop around $A_{i}$. Let $\hat{\pi}(S)$ be the minimal normal subgroup of $\pi_{f}(S)$ containing $g_{i}^{n_{i}}, i \in I$.

Definition 7. The corresponding covering $\hat{S}$ of $S$ is called the universal rational covering. If $\partial S \neq \varnothing$ we set $\hat{S}=(d S)^{\wedge}$.

For any polyhedron $S$ we denote by $\pi_{1}(S)$ the fundamental group of the underlying topological space. We say that $S$ is simply connected if $\pi_{1}(S)=1$.

THEOREM 1. Let $S$ be a compact connected rational polyhedral surface. Let $\alpha_{i}=\pi m_{i} / n_{i}$, $i=1, \ldots, M$ and $\alpha_{j}=2 \pi m_{j} / n_{j}, j=M+1, \ldots, N$ be the angles of boundary, respectively interior vertices of $S$.

(i) $\hat{S}$ is a closed connected simply connected non-compact developable polyhedral surface.

(ii) $\hat{S}$ with its canonical complex structure is isomorphic to $\mathbb{C}$ if and only if all the numerators $m_{i}=1, i=1, \ldots, N$. Otherwise $\hat{S}$ is isomorphic to the hyperbolic plane $\mathbb{H}$. The isomorphism $f: \hat{S} \rightarrow \mathbb{H}(\hat{S} \rightarrow \mathbb{C})$ carries the group $\pi_{r}(S)=\pi_{f}(S) / \hat{\pi}(S)$ of deck transformations of the covering $\hat{S} \rightarrow S$ into a discrete group $G$ of isometries of $H$ (respectively $\mathbb{C}$ ). If $S$ is oriented the projection $p: \hat{S} \rightarrow S$ is a covering of Riemann surfaces.

(iii) For any developing map $\varphi: \hat{S} \rightarrow \mathbb{C}$ there is a homomorphism $h: \pi_{r}(S) \rightarrow O(\mathbb{C})$ such that $\varphi$ is h-equivariant i.e. for any $g \in \pi_{r}(S), \varphi \circ g=h(g) \circ \varphi$. The developing map $\varphi$ is a holomorphic branched covering. The branching locus is contained in the set of vertices of $\hat{S}$. The branching number at a vertex $\hat{A} \in \hat{S}$ with angle $2 \pi m$ is $m$.

Proof. By proposition $1, \pi_{f}(\tilde{S})=1$ therefore $\pi_{1}(\tilde{S})=1$. The regular covering $q: \tilde{S} \rightarrow \hat{S}$ induces a homomorphism $q_{*}: \hat{\pi}(S) \rightarrow \pi_{1}(\hat{S}) \rightarrow 1$. The group $\hat{\pi}(S)$ is generated by conjugates of $g_{i}^{n_{i}}$ (taking $d S$ if necessary we can assume without loss of generality that $\partial S=\varnothing$ ). Each $g_{i}$ fixes some vertex at infinity of $\tilde{S}$, thus $\hat{\pi}(S)$ is generated by transformations with fixed points. Those generate the kernel of $q_{*}$, thus $\pi_{1}(\hat{S})=1$. Let $\hat{A}$ be a vertex of $\hat{S}$ above $A$ with angle $2 \pi m / n$. Going around $A$ once and lifting to $\hat{S}$ we rotate $\hat{S}$ about $\hat{A}$ by $2 \pi m / n$. Repeating it $n$ times we make a circle around $\hat{A}$, thus $\hat{A}$ has a finite number of adjacent faces and its angle is $2 \pi \mathrm{m}$.

The covering $\tilde{S}$ is developable almost by definition. Choose a reference face $P_{0}$ and an imbedding $\varphi_{0}: P_{0} \rightarrow \mathbb{C}$. Continue $\varphi_{0}$ analytically along a path $\gamma$ going from $P_{0}$ to some face $P$. The path $\gamma$ defines a face of $\tilde{P} \subset \tilde{S}$ above $P$. If $\gamma^{\prime}$ is another path homotopic to $\gamma$ we continue $\varphi_{0}$ analytically along the homotopy from $\gamma$ to $\gamma^{\prime}$ and conclude that the imbedding of $\tilde{P}$ depends only on the homotopy class of $\gamma$. Thus $\varphi_{0}$ uniquely continues to a developing map $\varphi: \tilde{S} \rightarrow \mathbb{C}$. Any automorphism $g$ of $\tilde{S}$ defines another developing map $\psi=\varphi \circ \mathrm{g}$. By proposition 2 , there is an isometry $h$ of $\mathbb{C}$ such that $\psi=h \circ \varphi$. Thus $h=h(g)$ is a homomorphism of $\pi_{f}(S)$ into $O(C)$ such that $\varphi$ is $h$-equivariant. Let $\hat{A} \in \tilde{S}$ be a vertex above $A \in S$ with angle $2 \pi m / n$. Let $g \in \pi_{f}(S)$ correspond to a simple loop around $A$. Then $h(g)$ is the rotation of $\mathbb{C}$ by $2 \pi m / n$ around $\varphi(\hat{A})$. Thus $h\left(g^{n}\right)=1$ therefore $\hat{\pi}(S) \subset \operatorname{Ker} h$. Therefore $\varphi: \tilde{S} \rightarrow$ 
$\mathbb{C}$ is invariant with respect to $\hat{\pi}(S)$ and it projects uniquely to a developing map of $S$. Since $S$ is developable it is not compact. We have now proved (i) and (iii).

Since the Riemann surface $\hat{S}$ is simply connected and non-compact, by the Riemann mapping theorem $\hat{S}$ is either $\mathbb{C}$ or $\mathbb{H}$. Denote both by $\mathrm{D}$ and let $f: \hat{S} \rightarrow D$ be an isomorphism. Any $g \in \pi_{r}(S)$ is an automorphism of $\hat{S}$, therefore it preserves (reverses) the canonical complex structure of $\hat{S}$ if $g$ preserves (reverses) the orientation. Thus $f$ induces an isomorphism of $\pi_{r}(S)$ onto a discrete subgroup $G$ of the group $O(D)$ of isometries of $D$ and identifies $S$ with the quotient $D / G$. If $S$ is oriented than $S=D / G$ as a Riemann surface.

It remains to prove that $D=\mathbb{C}$ if and only if $m_{i}=1, i=1, \ldots, N$. Doubling $S$ if necessary we assume that $\partial S=\varnothing$. Any polyhedral surface $S$ defines an orbifold ([11, ch. 13]). The orbifold $S$ has an Euler number $\chi_{0}(S)$ which is given by [11]

$$
\chi_{0}(S)=\chi(S)-\sum_{i=1}^{N}\left(1-1 / n_{i}\right)
$$

where $\chi(S)$ is the Euler characteristic of $S$. For any closed compact polyhedral surface $S$ with vertex angles $\alpha_{i}, i=1, \ldots, N$, we have

$$
\chi(S)=\sum_{i=1}^{N}\left(1-\alpha_{i} / 2 \pi\right)
$$

The proof of (3) is an elementary computation and is left to the reader. If $S$ is rational with vertex angles $2 \pi m_{i} / n_{i},(2)$ and (3) yield

$$
\chi_{0}(S)=\sum_{i=1}^{N}\left(1-m_{i}\right) / n_{i}
$$

Thus $\chi_{0}(S) \leq 0$ and the equality takes place if and only if $m_{i}=1$ for all $i$. Let $G_{0}$ be a subgroup of $G$ of finite index $n$ which acts freely on $D$. Then $D / G_{0}=R \rightarrow S$ is a covering with $n$ sheets and $\chi(R)=\chi_{0}(R)$. For a covering $R \rightarrow S$ of orbifolds with $n$ sheets we have $([11,13.3 .4])$

$$
\chi_{0}(R)=n \chi_{0}(S) .
$$

If $D=\mathbb{C}$ then $\chi(R)=0$ which is equivalent to $m_{i}=1, i=1, \ldots, N$.

Definition 8. A rational polyhedral surface is called flat (respectively hyperbolic) if the corresponding orbifold is isomorphic te $\mathbb{C} / G($ resp. $\mathbb{H} / G)$ for some discrete group $G$ of isometries.

The following corollary has been established in the course of the proof of theorem 1 (compare with [11, 13.3.6]).

Corollary 1. A rational polyhedral surface $S$ is flat if and only if the numerators of all vertex angles of $S$ are equal to 1 . Otherwise $S$ is hyperbolic.

COROLlary 2. Let $S$ be a polyhedral surface with boundary vertex angles $\pi m_{i} / n_{i}$, $i=1, \ldots, M$, and interior vertex angles $2 \pi m_{i} / n_{i}, i=M+1, \ldots, N$. Then the Euler number of the orbifold modelled on $S$ is

$$
\chi_{0}(S)=\frac{1}{2} \sum_{i=1}^{M}\left(1-m_{i}\right) / n_{i}+\sum_{i=M+1}^{N}\left(1-m_{i}\right) / n_{i}
$$


Proof. If $\partial S=\varnothing$ (6) becomes (4). If $\partial S \neq \varnothing$ (6) follows from (4) for $\mathrm{dS}$ and $\chi_{0}(d S)=2 \chi_{0}(S)$ by $(5)$.

From now on we identify $\hat{S}$ with $D(D=\mathbb{C}$ or $\mathbb{H})$ and the group $\pi_{r}(S)$ of the deck transformations of $\hat{S}$ with $G \subset O(D)$. We choose a developing map $\varphi: D \rightarrow \mathbb{C}$ and let $\Gamma \subset O(\mathbb{C})$ be the image of $G$ under the homomorphism $h: G \rightarrow O(\mathbb{C})$. Choose an origin in $\mathbb{C}$, let $C$ be the unit circle around it and let $O(C)$ be the group of isometries of $C$. Then $O(\mathbb{C})$ contains $O(C)$, the normal subgroup $\mathbb{C}$ of translations and $O(\mathbb{C})=O(C) \cdot \mathbb{C}$ is the semidirect product. Denote by $\bar{h}: G \rightarrow O(C)$ the composition of $h$ and the homomorphism $O(\mathbb{C}) \rightarrow O(C)$. Let $\bar{\Gamma}=\bar{h}(G) \subset O(C)$. For reasons that will become clear in $\S 2, h$ (resp. $\bar{h}$ ) is called the holonomy (resp. restricted holonomy) homomorphism. The group $\Gamma$ (resp. $\bar{\Gamma}$ ) is called the holonomy (resp. restricted holonomy) group of $S$.

Denote by $\mathbb{Z} / n \subset O(C)$ the group of rotations of order $n$ and by $D_{n} \subset O(C)$ the group generated by reflections in two axes meeting at the angle $\pi / n$. These groups exhaust all finite subgroups of $O(C)$.

PROPOSITION 3. Let $S$ be a compact rational polyhedral surface and let $n$ be the least common multiple of denominators of the vertex angles of $S$. Then $\breve{\Gamma}$ contains $\mathbb{Z} / n$. If $\partial S \neq \varnothing$ or if $S$ is not orientable then $\bar{\Gamma}$ contains $D_{n}$.

Proof. Let $\partial S=\varnothing$ and let $A_{i}, i=1, \ldots, N$, be the vertices of $S$ with angles $2 \pi m_{i} / n_{i}$. Let $g_{i} \in G$ be the simple loops around $A_{i}$. Then $h\left(g_{i}\right)$ is the rotation of $\mathbb{C}$ around $\varphi\left(A_{i}\right)$ by $2 \pi m_{i} / n_{i}$. Thus $\bar{h}\left(g_{i}\right)$ is a primitive rotation of order $n_{i}$. Together they generate $\mathbb{Z} / n$. Let $S$ be non-orientable and let $S^{\prime}$ be the orientable 2-sheeted covering of $S$. Then $G$ is generated by $G\left(S^{\prime}\right)$ and an element $r$ such that $r^{2} \in G\left(S^{\prime}\right)$. Then $h(r)$ reverses orientation so $\bar{h}(r)$ is a reflection. Thus $\bar{\Gamma}$ contains $\mathbb{Z} / \boldsymbol{n}$ and a reflection, so $D_{n} \subset \bar{\Gamma}$. If $\partial S \neq \varnothing$ then $G$ is generated by $G(d S)$ and an element $r$ such that $r^{2}=1$. Then $h(r)$ is a reflection therefore $D_{n} \subset \bar{\Gamma}$.

Definition 9. A rational polyhedral surface $S$ is called almost integrable if the restricted holonomy group $\bar{\Gamma}$ is finite.

In order to state proposition 4 we need the notion of the developing map along a path. Let $\gamma(t), 0 \leq t \leq 1$, be a piecewise differentiable path on a polyhedron $S$ going through faces $P_{1}, \ldots, P_{n}$. Any isometry $\varphi_{i}: P_{1} \rightarrow \mathbb{C}$ uniquely analytically continues to an immersion $\varphi: \bigcup_{i=1}^{n} P_{i} \rightarrow \mathbb{C}$ which is an isometry on each $P_{i}$. The mapping $\varphi$ is the developing map along $\gamma$.

Proposition 4. (i) A polygon $P$ is almost integrable if and only if the angle between any two sides of $P$ is $\pi$-rational.

(ii) Let a rational polyhedral surface $S$ be homeomorphic to the sphere with $n$ holes, $n=0,1, \ldots$ Let $Q_{1}, \ldots, Q_{n}$ be the connected components of $\partial S$. Choose a side $a_{i}$ of . $Q_{i}$ and $a$ path $\gamma_{i}$ from $a_{i}$ to $a_{i+1}, i=1, \ldots, n-1$. Then $S$ is almost integrable if and only if developing $S$ along $\gamma_{i}$ we map $a_{i}, a_{i+1}$ into intervals $a_{i}^{\prime}, a_{i+1}^{\prime}$ respectively with $a$ $\pi$-rational angle between them.

(iii) Let a rational polyhedral surface $S$ be homeomorphic to the projective plane $\mathbb{P}^{2}$. Then $S$ is almost integrable. 
Proof. The group $G$ is generated by simple loops $g_{i}$ around vertices, $\pi_{1}(S)$ and the flipping $r$ if $\partial S \neq \varnothing$. Proving proposition 2 we have shown that $\bar{h}\left(g_{i}\right), i=1, \ldots, N$, and $\bar{h}(r)$ generate a finite group $\bar{\Gamma}^{\prime} \subset \bar{\Gamma}$. In case (i), $\bar{h}\left(\pi_{1}(S)\right)=1$ and in case (ii), $\bar{h}\left(\pi_{1}(S)\right.$ is generated by rotations of finite order, thus $\bar{\Gamma}$ is finite. In case (iii) let $r$ be the generator of $\pi_{1}(S)$. Then $\bar{h}\left(g_{i}\right)$ generate $\mathbb{Z} / n$ and $\bar{h}(r)$ is a reflection, thus $\bar{\Gamma}=D_{n}$.

COROllary (of the proof). (i) Let $S$ be a polygon with $\pi$-rational angles between the sides and let $n$ be the least common multiple of denominators. Then $\bar{\Gamma}=D_{n}$.

(ii) Let $S$ be a rational polyhedral surface homeomorphic to $S^{2}$ (resp. $\mathbb{P}^{2}$ or the disc) and let $n$ be the least common multiple of denominators of the vertex angles. Then $\bar{\Gamma}=\mathbb{Z} / n\left(\right.$ resp. $\left.D_{n}\right)$.

Let $S$ be an almost integrable polyhedral surface. Denote by $G_{0}$ the kernel of $\bar{h}: G \rightarrow O(C)$ and by $R$ the quotient $D / G_{0}$. Then $R=S^{G_{0}}$ is the regular covering of $S$ with the group $\bar{\Gamma}$ of deck transformations. With this notation we have

THEOREM 2. (i) Let $n$ be the least common multiple of denominators of the vertex angles of $S$. Then $\bar{\Gamma}=\mathbb{Z} / n$ if $S$ is orientable and $\partial S=\varnothing$, otherwise $\bar{\Gamma}=D_{n}$, where $n^{\prime}$ is divisble by $n$.

(ii) $R$ is a compact Riemann surface without boundary and $G_{0}=\pi_{1}(R)$. The vertex angles of $R$ are multiples of $2 \pi$. The Euler characteristic $\chi(R)$ is equal to $n^{\prime} \chi_{0}(S)$ (resp. $2 n^{\prime} \chi_{0}(S)$ ) if $S$ is closed orientable (resp. otherwise) where $\chi_{0}(S)$ is the Euler number of $S$ given by (6).

(iii) The group $\Gamma_{0}=h\left(G_{0}\right)$ is a finitely generated group of parallel translations. The developing map $\varphi: D \rightarrow \mathbb{C}$ is h-equivariant, i.e. for any $g \in G_{0}$

$$
\varphi \circ g=h(g) \circ \varphi
$$

and $D / G_{0}=R$.

Proof. (i) By proposition 4, the group $\bar{\Gamma}$ is cyclic if $S$ is closed orientable and $\bar{\Gamma}$ is dihedral otherwise. By proposition $3, \bar{\Gamma}$ contains $\mathbb{Z} / n, D_{n}$ in the first, resp. second case.

(ii) If $g \in G$ has fixed points then $g$ has a fixed vertex $A$. Thus $h(g)$ fixes $\varphi(A)$ therefore $\bar{h}(g) \neq 1$. Analogously if $g$ reverses orientation then $h(g)$ does and $\bar{h}(g) \neq 1$. Thus $G_{0}$ acts freely by conformal automorphisms of $D$ inducing a complex structure on $R=D / G_{0}$ and $\pi_{1}(R)=G_{0}$. Besides $\chi(R)=\chi_{0}(R)=|\bar{\Gamma}| \chi_{0}(S)$ by (5). The vertex angles of $\hat{S}$ are multiples of $2 \pi$ and $G_{0}$ acts freely, thus vertex angles of $S / G_{0}$ are the same.

(iii) By definition $\bar{h}\left(G_{0}\right)=1$ so $\Gamma_{0} \subset \mathbb{C}$. If $p$ is the genus of $R$ then $G_{0}$ and therefore $\Gamma_{0}$ have $2 p$ generators. The rest is obvious.

The Riemann surface $R$ is called the canonical covering of an almost integrable surface $S$. In cases of particular interest we can calculate $\bar{\Gamma}$ and the genus $g(R)$ from the angles of $S$.

Proposition 5. (i) Let $\boldsymbol{S}$ be a polygon and let $\boldsymbol{n}$ be the least common multiple of the denominators of angles between sides of $S$. Then $\bar{\Gamma}=D_{n}$. Let $\pi m_{i} / n_{i}, i=1, \ldots, M$, be 
the vertex angles. Then

$$
g(R)=1+(n / 2) \sum_{i=1}^{M}\left(m_{i}-1\right) / n_{i}
$$

(ii) Let $S$ be homeomorphic to the disc and let $\pi m_{i} / n_{i}, i=1, \ldots, M,\left(2 \pi m_{i} / n_{i}\right.$, $i=M+1, \ldots, N)$ be the boundary (interior) vertex angles of $S$. Let $n$ be the least common multiple of $n_{i}$. Then $\bar{\Gamma}=D_{n}$ and

$$
g(R)=1+n\left[\frac{1}{2} \sum_{i=1}^{M}\left(m_{i}-1\right) / n_{i}+\sum_{i=M+1}^{N}\left(m_{i}-1\right) / n_{i}\right] .
$$

(iii) Let $S$ be homeomorphic to the sphere (resp. projective plane), let $2 \pi m_{i} / n_{i}$, $i=1, \ldots, N$, be the vertex angles and let $n$ be the least common multiple of $n_{i}$. Then $\bar{\Gamma}=\mathbb{Z} / n\left(\right.$ resp. $\left.\bar{\Gamma}=D_{n}\right)$ and

$$
g(R)=1+(n / 2) \sum_{i=1}^{N}\left(m_{i}-1\right) / n_{i}
$$

respectively

$$
g(R)=1+n \sum_{i=1}^{N}\left(m_{i}-1\right) / n_{i}
$$

Proof. The groups $\bar{\Gamma}$ were calculated in proposition 4. Using the fact that $2-2 g(R)=$ $\chi(R)=|\vec{\Gamma}| \chi_{0}(S)$ and formula (6) we obtain (8)-(11).

\section{Invariant surfaces of the billiard flow}

If a polygon $P$ is embedded in $\mathbb{C}$ we define the unit tangent bundle $T(P)$ to be the set of tangent vectors in $\mathbb{C}$ of length one with base points in $P$ and looking into $P$. The unit tangent bundle $T(S)$ of a polyhedral surface $S$ is made from $T\left(P_{i}\right)$ where $P_{i}$ runs over the faces of $S$, with obvious identifications.

The set $T(S)$ is the phase space of the geodesic flow on $S$ which is modelled on the movement of the billiard ball on $S$. The ball goes straight within each face. It bounces off boundary edges in an obvious way. Let the ball come to an edge $b$ between two faces $P$ and $Q$, at an interior point of $b$. We imbed $P \cup Q$ in $\mathbb{C}$ and let the ball cross straight from $P$ to $Q$. We agree to stop the ball at the vertices. We will see later that the trajectory has a natural continuation through a boundary (interior) vertex if and only if its angle is $\pi / n(2 \pi / n)$.

Definition 10. The flow on $T(S)$ defined above is the billiard flow of $S$.

If $x \in S$ is not a vertex, the fibre $T(S)_{x}$ is isomorphic to $C$ and the measure on $T(S)$ which is locally the product of Lebesgue measures on $\mathbb{C}$ and $C$ is preserved by the flow (cf. [9]). For any $x \in S$ the set of directions which will bring the ball from $x$ to a vertex is countable and therefore the set of $v \in T(S)$ which generate finite lifetime trajectories has measure 0 .

Let $x, y \in \dot{S}$ and let $\gamma$ be a piecewise differentiable curve on $S$ going from $x$ to $y$ and avoiding vertices. Developing $S$ on $\mathbb{C}$ along $\gamma$ we obtain an isomorphism $\left.T(S)\right|_{\gamma}=\gamma \times C$ and an isometry $T_{\gamma}: T(S)_{x} \rightarrow T(S)_{y}$ which depends on $\gamma$. It is called 
the parallel translation along $\gamma$. Moreover $T_{\gamma}$ depends only on the homotopy class $[\gamma] \in \pi_{f}(S)$.

From now on $S$ is assumed to be almost integrable. Choose a base point $x_{0} \in P_{0}$ and an imbedding $\varphi_{0}: P_{0} \rightarrow \mathbb{C}$. For any $x \in \dot{S}$ a choice of curve $\gamma$ from $x$ to $x_{0}$ gives an isometry $T_{\gamma}: T(S)_{x} \rightarrow C$. Varying $\gamma$ changes $T_{\gamma}$ by the action of $\bar{\Gamma}$ on $C$ which yields a mapping $\bar{\Theta}: T(\dot{S}) \rightarrow C / \bar{\Gamma}$. Now let $R$ be the canonical covering of $S$ and identify $P_{0}$ with a face of $R$. Since the restricted holonomy group of $R$ is trivial the construction above yields a mapping $\Theta: T(\dot{R}) \rightarrow C$. The action of $\bar{\Gamma}$ on $R$ obviously lifts to $T(R)$ and we have $T(S)=T(R) / \bar{\Gamma}$. The projection $p_{*}: T(R) \rightarrow T(S)$ of unit tangent bundles commutes with the billiard flows on $T(R)$ and $T(S)$, so the billiard on $S$ is the quotient of the billiard on $R$.

Proposition 6. (i) The mapping $\Theta(\bar{\Theta})$ uniquely extends to a continuous mapping $\Theta: T(R) \rightarrow C(\bar{\Theta}: T(S) \rightarrow C / \bar{\Gamma})$ invariant under the geodesic flow and such that the following diagram commutes

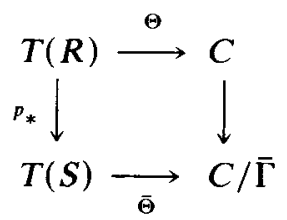

(ii) For $\theta \in C(\bar{\theta} \in C / \bar{\Gamma})$ denote by $R_{\theta} \subset T(R)\left(R_{\bar{\theta}} \subset T(S)\right)$ the set given by the equation $\Theta(v)=\theta(\bar{\Theta}(v)=\bar{\theta})$ and by $b_{\theta}^{\prime}\left(b_{\bar{\theta}}^{t}\right)$ the flow induced on $R_{\theta}\left(R_{\bar{\theta}}\right)$ by the billiard flow on $T(R)(T(S))$. Let $d: C \rightarrow C / \bar{\Gamma}$ be the projection and say that $\bar{\theta} \in C / \bar{\Gamma}$ is regular if any $\theta \in d^{-1}(\bar{\theta})$ has a trivial isotropy subgroup. Then

(a) for any $\theta \in C$ the projection $q: T(R) \rightarrow R$ induces a mapping $q_{\theta}: R_{\theta} \rightarrow R$ which is one-to-one everywhere except over vertices of $R$ with angles $2 \pi m>2 \pi$ where it is m-to-one;

(b) for any regular $\bar{\theta} \in C / \bar{\Gamma}$ and any $\theta \in d^{-1}(\bar{\theta})$ the projection $p_{*}$ induces an isomorphism of $R_{\theta}$ onto $R_{\bar{\theta}}$ which commutes with $b_{\theta}^{t}$ and $b_{\bar{\theta}}^{t}$ on $R_{\theta}$ and $R_{\bar{\theta}}$ respectively. Let $\theta \in C / \bar{\Gamma}$ be not regular and let $\bar{\Gamma}_{\theta}$ be the isotropy subgroup of $\theta \in d^{-1}(\bar{\theta})$. Then $\bar{\Gamma}_{\theta}$ acts on $R_{\theta}$ and $R_{\bar{\theta}}=R_{\theta} / \bar{\Gamma}_{\theta}$ with the flows $b_{\theta}^{t}$ and $b_{\bar{\theta}}^{t}$ respectively.

Proof. First of all we can define the billiard flow and the parallel translations on $T(\hat{S})$ where everything commutes with the projection $q_{*}: T(\hat{S}) \rightarrow T(R)$. Parallel translations on $T(\hat{S})$ define $\hat{\Theta}: T(\hat{S}) \rightarrow C$. Parallel translations and the geodesic flow on $T(\hat{S})$ are induced by the developing map $\varphi: \hat{S} \rightarrow \mathbb{C}$, thus $\hat{\Theta}$ is induced by the composition of $\varphi_{*}: T(\hat{S}) \rightarrow T(\mathbb{C})=\mathbb{C} \times C$ and the projection $\mathbb{C} \times C \rightarrow C$. Therefore $\hat{\Theta}$ is defined everywhere including the vertices, and commutes with the geodesic flow. All the mappings are compatible with the action of relevant groups, so we have the following commutative diagram

which implies (12).

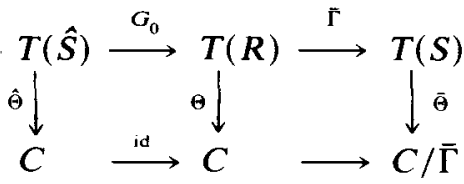


Fixing some $\theta \in C$ we have the constant vector field $X_{\theta}$ on $\mathbb{C}$ which is a crosssection of $T(\mathbb{C}) \rightarrow \mathbb{C}$. The set $\hat{S}_{\theta} \subset T(\hat{S})$ is the pullback of $X_{\theta}$ by $\varphi_{*}$. The branching properties of $\varphi$ imply (see theorem 1) that the projection $\hat{S}_{\theta} \rightarrow \hat{S}$ is one-to-one everywhere except above the vertices with angle $2 \pi m$ where it is $m$-to-1. Factoring out by the action of $G_{0}$ we obtain the same property for the projection $R_{\theta} \rightarrow R$. Commutative diagram (12) implies that for any $\theta \in C / \bar{\Gamma}$ the action of $\bar{\Gamma}$ on $T(R)$ permutes $R_{\theta_{i}}$ for $\theta_{i} \in d^{-1}(\bar{\theta})$ according to the action of $\bar{\Gamma}$ on $d^{-1}(\bar{\theta})$. The assertion (ii(b)) follows.

Proposition 6 shows that for regular $\bar{\theta} \in C / \bar{\Gamma}(C / \bar{\Gamma}$ has two irregular points if $\bar{\Gamma}$ is dihedral and none otherwise) the billiard flow on the invariant surface $R_{\bar{\theta}} \subset T(S)$ is equivalent to that on $R_{\theta} \subset T(R)$ for any $\theta \in d^{-1}(\bar{\theta})$. The projection $q_{\theta}: R_{\theta} \rightarrow R$ being essentially one-to-one, we use it to transfer the flows $b_{\theta}^{t}$ from $R_{\theta}$ to $R$, denote them by the same symbol and call them the billiard flows on $R$. It is clear from the construction that the flows $b_{\theta}^{t}$ have singularities at the vertices of $R$ with angles $2 \pi m>2 \pi$.

Proposition 7. (i) Let $\theta \in C$ be arbitrary. Let $l_{\theta}^{t}$ be the linear flow on $\mathbb{C}$ in direction $\theta$, i.e. $l_{\theta}^{t} z=z+t e^{i \theta}$. Let $\varphi_{*}^{-1} l_{\theta}^{t}$ be the pullback of $l_{\theta}^{t}$ on $\hat{S}$ by the developing map $\varphi: \hat{S} \rightarrow \mathbb{C}$. Then the flow $\varphi_{*}^{-1} l_{\theta}^{t}$ is invariant under the action of $G_{0}$ and the induced flow on $R=D / G_{0}$ is $b_{\theta}^{t}$.

(ii) Each vertex of $R$ with angle $2 \pi m>2 \pi$ is a singular point of $b_{\theta}^{t}$ with uniformly spaced $m$ incoming and $m$ outgoing separatrices. Other points of $R$ are non-singular. The flows $b_{\theta}^{t}$ are obtained from any one of them by rotation.

(iii) The billiard flow on $T(R)$ is isomorphic to $R \times C$ with the flow $b_{\theta}^{t}$ on $R \times \theta$.

Proof. From the proof of proposition 6 we see that the geodesic flow on $\hat{S}$ is induced by the developing map $\varphi: \hat{S} \rightarrow \mathbb{C}$. Proposition 7 follows easily from that, the information about the branching of $\varphi$ (theorem 1) and the fact that $h\left(G_{0}\right)=\Gamma_{0}$ is a group of translations, thus it leaves $l_{\theta}^{t}$ invariant.

Recall that a flow $b^{t}$ on a manifold $M$ smooth everywhere except at a finite number of multisaddle singular points is called minimal (quasiminimal in [13]) if every infinite semi-trajectory of $b^{t}$ is dense in $M$.

Definition 11. A direction $\theta$ is called rational with respect to a set $X \subset \mathbb{C}$ (with respect to a group $\Gamma$ of translations) if there exists a straight line in direction $\theta$ which meets $X$ in two points (which contains points $z_{1}, z_{2}$ such that the vector $z_{1}-z_{2} \in \Gamma$ ).

Proposition 8. Let $S, R, \Gamma_{0}$ and $b_{\theta}^{t}$ be as before. Denote by $V \subset \mathbb{C}$ the image of the branching locus of $\varphi: D \rightarrow \mathbb{C}$. Then the flow $b_{\theta}^{t}$ has a periodic trajectory (resp. is not minimal) only if $\theta$ is rational with respect to $\Gamma_{0}$ (resp. $\theta$ is rational with respect to $V$ ). Proof. Let $\gamma(t)$ be a periodic trajectory of $b_{\theta}^{t}$ with period $T$. Then the lifting $\hat{\gamma}(t)$ on $D$ has the property $\hat{\gamma}(t+T)=g \hat{\gamma}(t)$ for some $g \in G_{0}$. The image $\varphi_{*} \hat{\gamma}(t)$ is the straight line in direction $\theta$, thus the equivariance of $\varphi$ implies that $\theta$ is rational with respect to $\Gamma_{0}$. Let $b_{\theta}^{t}$ have a non-dense infinite semi-trajectory. Arguing as in [3] or as in [13] (where theorem 9 of [6] is used) we conclude that $b_{\theta}^{t}$ has trajectory $\gamma$ 
going from one singular point to another. Thus both ends of $\hat{\gamma}$ are vertices of $\hat{\boldsymbol{S}}$ and $\varphi(\hat{\gamma})$ is a straight interval with both ends in $V$.

COROLlary. The flow $b_{\theta}^{t}$ is not minimal for at most a countable set of $\theta$ 's.

Proof. The set of branching points of $\varphi: S \rightarrow \mathbb{C}$ is at most countable.

\section{Ergodicity and spectrum}

We keep the notation of the previous sections. We will assume that the reader is familiar with the basic notions of ergodic theory (cf. [9]). The flow $b_{\theta}^{t}$ preserves the Lebesgue measure on $R$ and let $U_{\theta}^{t}$ be the corresponding group of unitary operators on $L_{2}(R)$. For any discrete group $L$ of translations the linear flow $l_{\theta}^{t}$ defines a flow on the torus $T=\mathbb{C} / L$ denoted by the same symbol.. When $L$ is fixed we call $\theta \in C$ rational if it is rational with respect to $L$. It is well known that the flow $l_{\theta}^{t}$ on the torus is ergodic if and only if it is minimal if and only if $\theta$ is an irrational direction. Moreover for irrational $\theta$ the flow is uniquely ergodic and for rational $\theta$ it is periodic. The spectrum of $l_{\theta}^{t}$ is discrete. We will generalize these facts to a certain class of billiards.

Denote by $V_{0} \subset V$ the set of fixed points of rotations in $\Gamma$. Any two points $x, y \in \mathbb{C}$ define a translation vector $y-x$.

Definition 12. A point $z \in \mathbb{C}$ is called rational if there exists $z_{0} \in V_{0}$ and $a_{1}, a_{2} \in \Gamma_{0}$ such that $z-z_{0}=r_{1} a_{1}+r_{2} a_{2}$ for some rational numbers $r_{1}, r_{2}$.

THEOREM 3. Let $S$ be an almost integrable polyhedral surface such that the monodromy group $\Gamma \subset O(\mathbb{C})$ is discrete and such that all points of $V$ are rational. Then:

(i) The following are equivalent:

(a) direction $\theta$ is irrational;

(b) flow $b_{\theta}^{t}$ is minimal;

(c) flow $b_{\theta}^{t}$ is ergodic with respect to the Lebesgue measure;

(d) $b_{\theta}^{t}$ is uniquely ergodic.

(ii) Let $l_{\theta}^{t}$ be the linear flow on $T=\mathbb{C} / \Gamma_{0}$. For every irrational direction $\theta$ the discrete spectrum of $b_{\theta}^{t}$ coincides with the spectrum of $l_{\theta}^{t}$. The continuous spectrum of $b_{\theta}^{t}$ is empty if and only if $S$ is flat.

(iii) For every rational $\theta$ the flow $b_{\theta}^{t}$ is periodic.

Proof. If $\Gamma_{0}$ is discrete, theorem 2(iii) implies that there is a commutative diagram

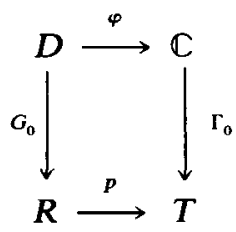

that yields a branched covering $p: R \rightarrow T$ which projects $b_{\theta}^{t}$ on $l_{\theta}^{t}$.

It is well known [2] that there are 17 types of discrete groups $\Gamma \subset O(\mathbb{C})$. The set $V_{0}$ of centres of rotations together with the axes of reflections and sliding reflections of $\Gamma$ form a pattern of lines and points in $\mathbb{C}$. Choose two generators $a, b$ of $\Gamma_{0}$ and 
take some $0 \in V_{0}$ for the origin in $\mathbb{C}$. An obvious choice of coordinates $x, y$ identifies $a$ with $(1,0), b$ with $(0,1)$ and $\Gamma_{0}$ with the integer lattice. It is easy to verify case by case that every $A \in V_{0}$ has rational coordinates and that every axis of (sliding) reflection has two points with rational coordinates. By assumption all points of $V$ have rational coordinates. Observe that all points of $V \backslash V_{0}$ come from (boundary) vertices of $S$ with angle $(\pi m>\pi) 2 \pi m>2 \pi$ and that $V$ is invariant under the action of $\Gamma_{0}$. There is a finite number of orbits of $V$ under the action of $\Gamma_{0}$, therefore coordinates of $z \in V$ have a common denominator, say $N$. Let $\Gamma_{0}^{\prime}$ be the group generated by $a / N$ and $b / N$, denote by $x, y$ the new coordinates and by $Q=$ $\{0 \leq x, y \leq 1\}$ the fundamental parallelogram of $\Gamma_{0}^{\prime}$. Now the points of $V$ have integer coordinates and the whole plane is the union of

$$
Q_{m, n}=\{m-1 \leq x \leq m, n-1 \leq y \leq n\} .
$$

Denote the torus $\mathbb{C} / \Gamma_{0}^{\prime}$ by $T_{1}$ and let $p^{\prime}: T \rightarrow T_{1}$ and $p_{1}=p^{\prime} p: R \rightarrow T_{1}$ be the natural projections. The covering $p_{1}$ is branched only over $(0,0) \in T_{1}$, therefore $R$ can be represented as a union of $Q_{i}, i \in I$, such that $p_{1}: Q_{i} \rightarrow Q$ is 1-to-1 for all $i$. Choose a fundamental domain $\tilde{R} \subset D$ for $R$, then $\tilde{R}=\bigcup_{I} Q_{i}$. Since the composition of $\varphi: D \rightarrow$ $\mathbb{C}$ and the projection $\mathbb{C} \rightarrow T_{1}$ maps each $Q_{i}$ onto $T_{1}, \varphi$ must map $Q_{i}$ on some $Q_{m, n}$ for all $i \in I$. Thus we have represented $R$ by the union of a finite number of $Q_{m, n}$ where each $Q_{m, n}$ may be taken with some multiplicity. Let us call this object a polygon in the lattice $\Gamma_{0}^{\prime}$ and denote it by $R^{\prime}$. Identifications on the boundary of $R^{\prime}$ are made by elements of $\Gamma_{0}$.

This construction represents the billiard flow $b_{\theta}^{t}$ on $R$ by the linear flow in the direction $\theta$ on $R^{\prime}$. When the ball reaches a boundary edge of $R^{\prime}$ it gets transferred by some $g \in \Gamma_{0}$ to another edge and then it keeps rolling in the same direction. We think of $Q_{i}, i \in I$, as parallelograms on the plane and let $X$ be the union of their bases. Thus $X=[0,1) \times I$. The flow $b_{\theta}^{t}$ that started on the base of some $Q_{i}$ reaches, after a certain lapse of time $T_{\theta}$, the tops of two parallelograms of $R^{\prime}$ which are identified with the bases of, say, $Q_{j}$ and $Q_{j^{\prime}}$ (see figure 2). Every point of the base of $Q_{i}$ comes to its destination translated by the rotation number $\alpha$ of the flow $l_{\theta}^{t}$. Also the $[0,1-\alpha)$ part of $Q_{i}$ comes to $Q_{j}$ and the $[1-\alpha, 1)$ part of $Q_{i}$ comes to $Q_{j^{\prime}}$. Denote by $\rho_{\alpha}$ the rotation of $[0,1)$ by $\alpha$. The previous remarks can be summarized as follows. There exists a function $w(x)$ on $[0,1)$ with values in the permutation group $\sum_{I}$ of $I$ symbols, constant on $[0,1-\alpha)$ and $[1-\alpha, 1)$, so that the first return to $X$ map $\tau_{\alpha}$ is

$$
\tau_{\alpha}(x, i)=(x+\alpha, w(x) i) \quad \text { for } x \in[0,1), i \in I .
$$

Thus $\tau_{\alpha}$ is the extension of rotation $\rho_{\alpha}$ with the skewing function $w(x)$. The flow $b_{\theta}^{t}$ is the suspension of $\tau_{\alpha}$ with the constant time of return function. To show (i) we use an unpublished result of W. Veech.

THEOREM (W. Veech). Any extension $\tau_{\alpha}$ of an irrational rotation $\rho_{\alpha}$ with the skewing function constant on $[0,1-\alpha)$ and $[1-\alpha, 1)$ is ergodic if and only if it is minimal. The ergodic components of $\tau_{\alpha}$ are in 1-to-1 correspondence with the orbits on $I$ of the group $W \subset \sum_{I}$ generated by $w([0,1-\alpha))$ and $w([1-\alpha, 1))$. 


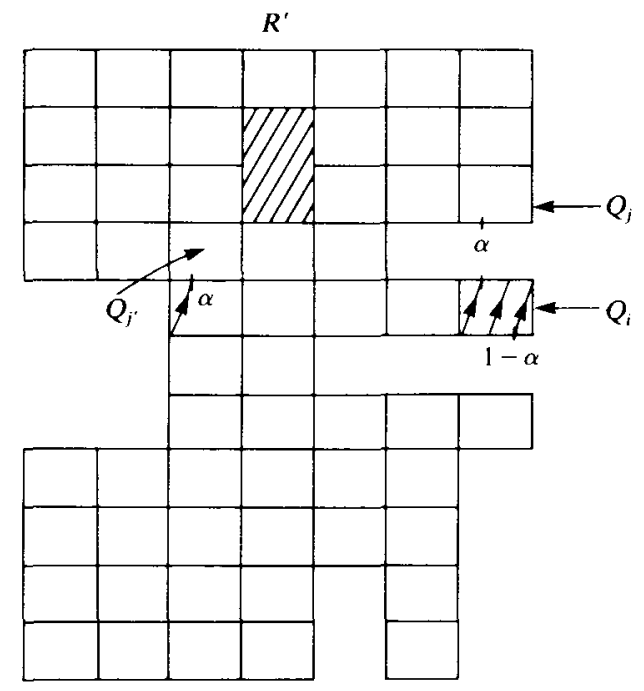

FIGURE 2. Boundary edges are identifled by parallel translations. The shaded area does not belong to $R^{\prime}$, it comes from an obstacle.

Ergodicity or minimality of $\tau_{\alpha}$ is equivalent to the ergodicity or minimality of $b_{\theta}^{t}$. If the direction $\theta$ is irrational then $b_{\theta}^{t}$ is minimal by proposition 8 and $\alpha$ is irrational. Thus by Veech's theorem, $\tau_{\alpha}$ is ergodic. For finite extensions of irrational rotations ergodicity is equivalent to unique ergodicity which proves (i). If $\theta$ is rational then $l_{\theta}^{t}$ is periodic and $b_{\theta}^{t}$ being a finite extension of it is too.

Pulling back by $p: R \rightarrow T$ we imbed $L_{2}(T)$ isometrically into $L_{2}(R)$ as functions constant on the fibres. We want to show that $L_{2}(R) \ominus L_{2}(T)$ does not contain eigenfunctions of $b_{\theta}^{t}$. Every nontrivial eigenfunction of $b_{\theta}^{t}$ comes from an eigenfunction $f(x, i)$ of $\tau_{\alpha}$ with an eigenvalue $\lambda$. Then $g(x, i, j)=f(x, i) \bar{f}(x, j)$ is a fixed function of the transformation $\tau_{\alpha}^{\prime}$ of $[0,1) \times I \times I$ given by

$$
(x, i, j) \rightarrow(x+\alpha, w(x) i, w(x) j) .
$$

Thus by Veech's theorem, $g(x, i, j)=h(i, j)$ where $h(w i, w j)=h(i, j)$ for any $w \in W$. Since $\tau_{\alpha}$ is minimal, $W$ acts transitively on $I$ and let $W_{0}$ be the isotropy subgroup of 1 . Set $h(i)=h(1, i)$, then $h$ is $W_{0}$-invariant, $|h|=1$ and

$$
f(x, i)=h(i) f(x, 1)
$$

Applying $\tau_{\alpha}$ to both sides of (15) we get

$$
f(x+\alpha, w(x) i)=h(w(x) i) f(x+\alpha, 1)=\lambda f(x, i)=\lambda h(i) f(x, 1) .
$$

Thus for each generator $w \in W$ there is an interval $J \subset[0,1)$ such that for all $x \in J$

$$
h(w i) h(i)^{-1}=\lambda f(x, 1) f(x+\alpha, 1)^{-1} \text {. }
$$

Since the right hand side of (16) does not depend on $i$, there is $c(w) \in C$ such that

$$
h(w i)=c(w) h(i)
$$

for all $i \in I$. It follows from (17) that $w \rightarrow c(w)$ is a character of $W$ trivial on $W_{0}$. Its kernel $W_{1}$ is a normal subgroup containing $W_{0}$. Let $m=\left[W: W_{1}\right]$ and let $I_{1} \subset I$ 
be the $W_{1}$-orbit of 1 . Then the character $c$ induces an isomorphism $c: W / W_{1} \rightarrow \mathbb{Z} / m$, the group $W_{1}$ has $m$ orbits $I_{1}, \ldots, I_{m}$ of the same magnitude $|I| / m$, and $\mathbb{Z} / m$ permutes them cyclically. Therefore if we represent elements of $I$ by pairs $(i, j) 1 \leq i \leq m$, $1 \leq j \leq|I| / m$ we have

$$
\tau_{\alpha}(x, i, j)=\left(x+\alpha, c(w(x)) i, w_{1}(x, i) j\right) .
$$

The transformation $\sigma_{\alpha}:(x, i) \rightarrow(x+\alpha, c(w(x)) i)$ is an $m$-point extension of $\rho_{\alpha}$ and $\tau_{\alpha}$ is a $|I| / m$-point extension of $\sigma_{\alpha}$. The eigenfunction $f$ of $\tau_{\alpha}$ is the pullback of an eigenfunction $\tilde{f}$ of $\sigma_{\alpha}$ and $\sigma_{\alpha}$ is equivalent to the rotation by $\alpha$ of $[0, m)$.

Going back to the flows we conclude that there is a torus $\dot{T}$, a branched covering $\tilde{p}: R \rightarrow \tilde{T}$ with $|I| / m$ sheets and a covering $q: \tilde{T} \rightarrow T_{1}$ (unbranched) with $m$ sheets such that $p_{1}=q \tilde{p}$ and the diagram below commutes

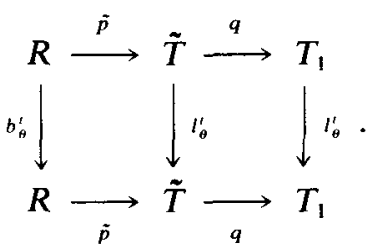

Thus we know that any eigenfunction of $b_{\theta}^{t}$ is the pullback from some torus $\tilde{T}$. Let $\tilde{T}=\mathbb{C} / \tilde{\Gamma}_{0}$. Since $R$ is the polygon $\tilde{R}$ with sides identified by some $g_{i}$, the group $\tilde{\Gamma_{0}}$ must contain all $g_{i}$. But $g_{i}$ generate $\Gamma_{0}$, thus $\Gamma_{0} \subset \tilde{\Gamma}_{0}$ which implies that the covering $\tilde{p}: R \rightarrow \tilde{T}$ factors through $p$ and some $\tilde{q}: T \rightarrow \tilde{T}$ where all the mappings commute with the relevant flows. This argument shows that $T$ is the maximal torus covered by $R$. Therefore we can pull an eigenfunction back from $\tilde{T}$ to $R$ in two steps: first from $\tilde{T}$ to $T$ and then from $T$ to $R$. The continuous spectrum of $b_{\theta}^{t}$ is empty if and only if $L_{2}(R)=L_{2}(T)$ which means that $p: R \rightarrow T$ is an isomorphism.

Corollary 1. Let $P$ be a polygon with $\pi$-rational angles between the sides. Assume that the obstacles of $P$ have no slits. If the group $\Gamma$ generated by reflections in the sides of $P$ is discrete then the billiard flow $b_{\theta}^{t}$ is uniquely ergodic for any irrational direction $\theta$.

Proof. Since $P$ has no vertices with angles $\pi m>\pi$, we have $V=V_{0}$. Points of $V_{0}$ are rational with respect to $\Gamma$ by definition, therefore assumptions of theorem 3 are satisfied.

COROllary 2. Let $P$ be a polygon with $\pi$-rational angles between sides such that the group $\Gamma$ generated by reflections in the sides of $P$ is discrete. Let $A$ be a vertex of a slit and continue the line of the slit until it crosses $a$ side $b$ of $P$. Let $B$ be the point of intersection. If the vector $B-A$ is rational with respect to $\Gamma$ (for every slit in $P$ ) then $b_{\theta}^{\prime}$ is uniquely ergodic for any irrational direction $\theta$.

Proof. The slit and the side $b$ define axes of reflections in $\Gamma$. It was mentioned in the proof of theorem 3 that those are rational lines. The intersection of rational lines is a rational point. Since $B$ is rational and $B-A$ is by assumption, $A$ is rational and we are able to apply theorem 3 . 
COROLlARY 3. Let $S$ be a rational polyhedral surface homeomorphic to the sphere or the disc. Assume that $S$ has no boundary (interior) vertices with angles $\pi m(2 \pi m), m>$ 1. Let the holonomy group $\Gamma$ of $S$ be discrete. Then for any irrational direction $\theta$ the billiard flow $b_{\theta}^{t}$ is uniquely ergodic.

Proof. By proposition 4(ii), $S$ is almost integrable. Apply theorem 3.

Corollary 4. Let $S$ be any Platonic solid except the dodecahedron. Then the billiard flow on $S$ in any irrational direction is uniquely ergodic.

Proof. Developing $S$ on the plane one sees that $\Gamma$ is not discrete only for the dodecahecron.

\section{REFERENCES}

[1] B. Eckhardt, J. Ford \& F. Vivaldi. Analytically solvable dynamical systems which are not integrable. Preprint, 1983.

[2] E. S. Fedorov, Zap. Imp. S. Petersburg Mineral. OBSC. 28 (1971), 345-390 (in Russian).

[3] R. H. Fox \& R. B. Kershner. Concerning the transitive properties of geodesics on a rational polyhedron. Duke Math. J. 2 (1936), 147-150.

[4] E. Gutkin. Geodesic flows on rational polyhedra. Preprint, 1982.

[5] H. Masur. Interval exchange transformations and measured foliations. Ann. Math. 115 (1982), 169-200.

[6] A. Mayer. Trajectories on the closed orientable surfaces. Mat. Sbornik 12 (1943), 71-84 (in Russian).

[7] P. T. Richens \& M. V. Berry. Pseudo-integrable systems in classical and quantum mechanics. Physica 2D (1981), 495-512.

[8] C. Rodenberg. Geodatische Linien auf Polyederflächen. Rend. Circ. Mat. Palermo 23 (1907), 107-125.

[9] Y. Sinai. Introduction to Ergodic Theory. Princeton University Press: Princeton, 1976.

[10] P. Stäckel. Geodatische Linien auf Polyederflächen. Rend. Circ. Mat Palermo 22 (1906), 141-151.

[11] W. Thurston. The geometry and topology of three-manifolds. Mimeographed notes: Princeton, 1980.

[12] W. A. Veech. Gauss measures for transformations on the space of interval exchange maps. Ann. Math. 115 (1982), 201-242.

[13] A. N. Zemlyakov \& A. B. Katok. Topological transitivity of billiards in polygons. Matem. Zametki 18:2 (1975), 291-300 (in Russian; English transl. in Mat. Notes 18:2 (1976), 760-764). 